\title{
07
}

\section{Электронная структура наноинтерфейса Cs/n-GaN(0001)}

() Г.В. Бенеманская ${ }^{1}$, М.Н. Лапушкин ${ }^{1,9}$, Д.Е. Марченко ${ }^{2}$, С.Н. Тимошнев ${ }^{3}$

${ }^{1}$ Физико-технический институт им. А.Ф. Иоффе РАН, Санкт-Петербург, Россия

${ }^{2}$ Helmholtz-Zentrum Berlin für Materialien und Energie, Elektronenspeicherring BESSY II, Berlin, Germany

${ }^{3}$ Санкт-Петербургский национальный исследовательский академический университет РАН, Санкт-Петербург, Россия

ฯ E-mail: Lapushkin@ms.ioffe.ru

\section{Поступило в Редакцию 23 мая 2017 г.}

Впервые проведены фотоэмиссионные исследования методом фотоэлектронной спектроскопии in situ электронной структуры поверхности $n-\mathrm{GaN}(0001)$ и интерфейса $\mathrm{Cs} / n-\mathrm{GaN}(0001)$ в диапазоне субмонослойных Cs-покрытий. Изучены спектры фотоэмиссии из валентной зоны, спектры поверхностных состояний и остовных уровней $\mathrm{Ga} 3 d$, Cs $4 d$ и Cs $5 p$ при синхротронном возбуждении в диапазоне энергий фотонов $50-150 \mathrm{eV}$. Обнаружена эволюция спектра поверхностных состояний вблизи максимума валентной зоны при адсорбции атомов Cs. Показан металлический характер наноинтерфейса $\mathrm{Cs} / n-\mathrm{GaN}(0001)$.

DOI: $10.21883 /$ PJTF.2018.06.45767.16885

Широкозонные полупроводники нитриды III группы представляют большой интерес благодаря их интенсивному использованию в оптоэлектронных приборах, работающих в широком спектральном диапазоне от инфракрасного до ультрафиолетового. $\mathrm{GaN}$ также является наиболее перспективным материалом для создания новых электронных устройств на основе наногетероструктур, работающих в агрессивных средах, при повышенных температурах и мощностях.

Наиболее полную информацию об электронной структуре дают результаты фотоэмиссионных исследований с помощью фотоэлектронной спектроскопии. Электронная структура $\mathrm{GaN}(0001)$ довольно подробно исследована экспериментально [1-6] и теоретически [7-11]. Спектр фотоэмиссии в области валентной зоны представляет собой 
слабоструктурированную полосу шириной $\sim 9 \mathrm{eV}$ ниже края валентной зоны $E_{\mathrm{VBM}}$ с некоторыми особенностями в спектре, природа которых, как правило, связана с поверхностными состояниями [7,9]. Спектр поверхностных состояний зависит от различных атомных структур на $\mathrm{Ga-полярной} \mathrm{поверхности} \mathrm{GaN}(0001)$. Например, для $\mathrm{GaN}(0001)-2 \times 2$ в точке Г были обнаружены поверхностные состояния вблизи $E_{\mathrm{VBM}}$ в запрещенной зоне, а для $\mathrm{GaN}(0001)-1 \times 1$ в точке $\Gamma$ таких состояний в запрещенной зоне не найдено [2,3]. Следует отметить, что энергетическое положение поверхностного состояния вблизи $E_{\mathrm{VBM}}$ зависит от подготовки образца [4].

Проведенные расчеты электронной структуры [7-11] для различных реконструкций $\mathrm{GaN}(0001)$ показывают наличие в Г-точке спектра поверхностных состояний, природа которых связана с $\mathrm{N} p$ - и $\mathrm{Ga} s-p$-орбиталями оборванных связей. Так, в [8] для поверхности $\mathrm{GaN}(0001)-2 \times 2$ рассчитано распределение плотности поверхностных состояний оборванных связей атомов $\mathrm{Ga}$ и получено, что в запрещенной зоне существуют заполненные поверхностные состояния.

Электронная структура интерфейса $\mathrm{Cs} / n-\mathrm{GaN}(0001)$ до сих пор недостаточно изучена. В основном исследования были направлены на получение подложек $\mathrm{Cs} / \mathrm{O} / p-\mathrm{GaN}(0001)$ с малым отрицательным электронным сродством $[12,13]$. Было показано, что адсорбция Cs на $p$ $\mathrm{GaN}(0001)$ приводит к сильному изгибу зон и подавлению собственных поверхностных состояний подложки [14]. Поверхностные состояния вблизи уровня Ферми $\left(E_{\mathrm{F}}\right)$, индуцированные адсорбцией Cs были найдены методом пороговой фотоэмиссионной спектроскопии [15].

Существует ряд расчетов адсорбции Cs на GaN $[10,11,16,17]$. При адсорбции Cs на $n$ - $\mathrm{GaN}(0001)-2 \times 2$ показано, что при покрытиях 0.33 монослоя (ML) происходит формирование зоны около $E_{\mathrm{F}}$, образованной при гибридизации орбиталей Cs $6 s$, Cs $5 p$ и Ga $4 p$ [10]. Других состояний в области валентной зоны не найдено. Показано, что при покрытиях $\theta=0.33 \mathrm{ML}$ разница между энергиями связи при адсорбции Cs на различных адсорбционных местах незначительна. Однако более предпочтительна адсорбция Cs на позициях $B_{\mathrm{N}}$ (мостиковая позиция между атомами $\mathrm{N}$ ) и $\mathrm{H}_{3}$ (hollow, ямочная позиция). В этом случае энергия связи адсорбции составляет $2.04 \mathrm{eV}$. Увеличение Cs-покрытия до $1 \mathrm{ML}$ приводит к уменьшению энергии адсорбции до $0.5 \mathrm{eV}$, и наиболее предпочтительной становится позиция $B_{\mathrm{N}}$.

4* Письма в ЖТФ, 2018, том 44, вып. 6 
В настоящей работе впервые проведены исследования фотоэмиссии наноинтерфейса $\mathrm{Cs} / n-\mathrm{GaN}(0001)$ при субмонослойных покрытиях в широком диапазоне энергий возбуждения от 50 до $150 \mathrm{eV}$, что позволило изучить фотоэмиссию и электронную структуру поверхностного слоя $n-\mathrm{GaN}(0001)$ и наноинтерфейса $\mathrm{Cs} / n-\mathrm{GaN}(0001)$, а также определить характер интерфейса.

Фотоэмиссионные исследования были выполнены на российскогерманском канале синхротрона BESSY II (Берлин, Германия) с использованием метода фотоэлектронной спектроскопии при возбуждении в диапазоне энергий фотонов $50-150 \mathrm{eV}$. Регистрировались фотоэлектроны по нормали к поверхности, возбуждающий пучок падал на поверхность образца под углом $45^{\circ}$. Исследования образцов $n-\mathrm{GaN}(0001)$ и интерфейса $\mathrm{Cs} / n-\mathrm{GaN}(0001)$ проводились in situ в вакууме $P<5 \cdot 10^{-10}$ Torr при комнатной температуре. Регистрировались спектры в области валентной зоны, спектры поверхностных состояний, a также спектры остовных уровней $\mathrm{Ga} 3 d$, Cs $4 d$ и Cs $5 p$. Полное энергетическое разрешение составляло $50 \mathrm{meV}$.

Образец $n$ - $\mathrm{GaN}(0001)$ толщиной $\sim 4 \mu \mathrm{m}$ выращен методом осаждения из металлоорганических соединений на сапфировой подложке и легирован $\mathrm{Si}\left(\sim 4 \cdot 10^{17} \mathrm{~cm}^{-3}\right)$. Образец имел отрелаксированную систему протяженных дефектов с когерентным согласованием доменов мозаичной структуры и образованием дилатационных границ с низкой плотностью дислокаций $\sim 10^{8} \mathrm{~cm}^{-2}$ и подвижностью электронов $\sim 600 \mathrm{~cm}^{2} \cdot \mathrm{V}^{-1} \cdot \mathrm{s}^{-1}$. Ширина запрещенной зоны $E_{g}=3.4 \mathrm{eV}$. Данные рентгеновского дифракционного анализа указывают на высокоупорядоченную структуру в слоях $\mathrm{GaN}$. Поверхностная морфология образцов исследована с помощью атомно-силовой микроскопии (ACM). Данные АСМ свидетельствуют об атомарно-гладкой поверхности образцов $\mathrm{GaN}$ при среднеквадратичной шероховатости $\sim 1 \mathrm{~nm}$.

Перед фотоэмиссионными исследованиями образцы подвергались отжигу in situ при температуре $\sim 900 \mathrm{~K}$. Атомарно-чистый Cs напылялся пошагово на чистую поверхность образца из стандартного источника. Отметим, что за один монослой Cs принимается концентрация $5.2 \cdot 10^{14}$ atom $/ \mathrm{cm}^{2}$, при которой формируется укомплектованный слой атомов Cs [18].

Нормированные спектры фотоэмиссии в области валентной зоны $n-\mathrm{GaN}(0001)$ в диапазоне энергий возбуждения $50-150 \mathrm{eV}$ подробно представлены на рис. 1. Спектры приведены к энергии вершины 


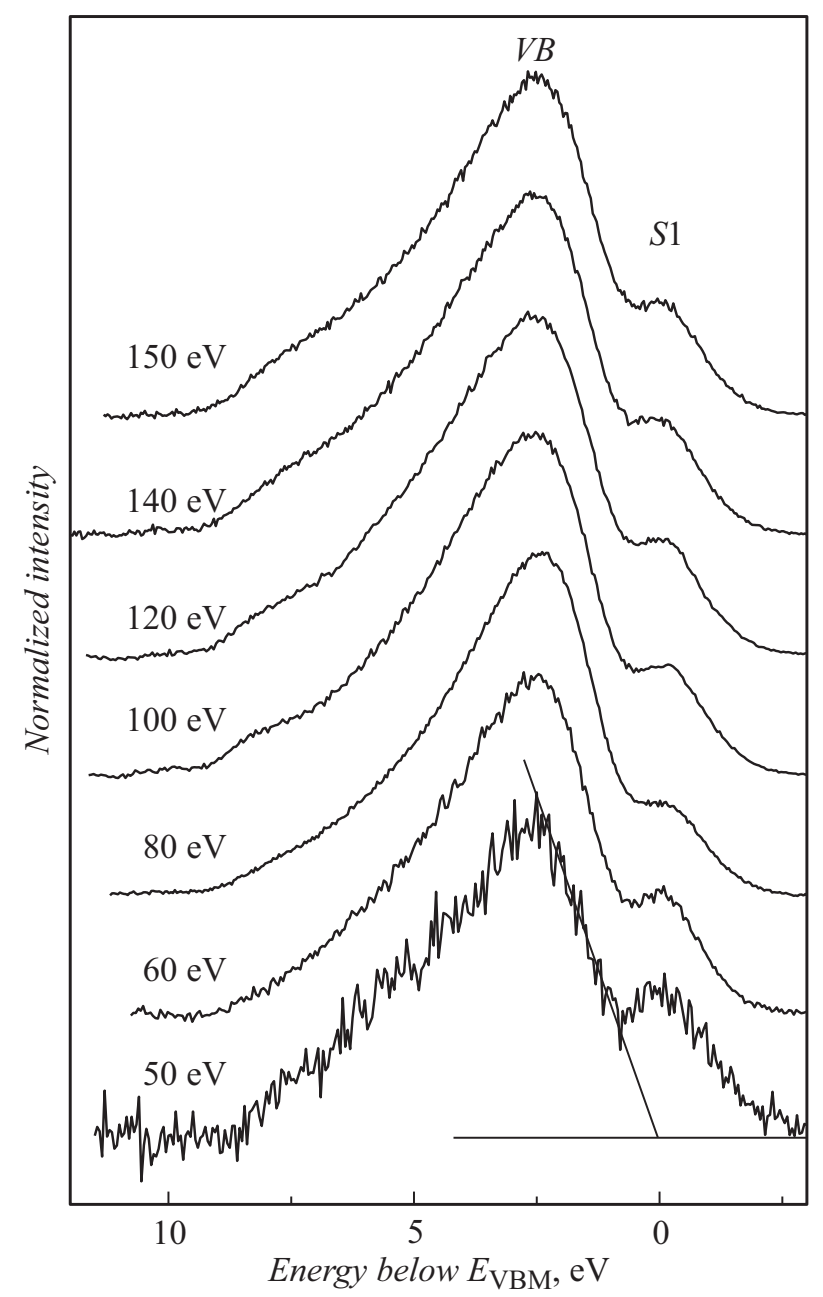

Рис. 1. Нормированные спектры фотоэмиссии в области валентной зоны для чистой поверхности $n$ - $\mathrm{GaN}(0001)$ при различных энергиях возбуждения $h v$ от 50 до $150 \mathrm{eV}$. Для энергии возбуждения $h v=50 \mathrm{eV}$ приведена линейная аппроксимация пика валентной зоны для определения положения $E_{\mathrm{VBM}}$.

Письма в ЖТФ, 2018, том 44, вып. 6 

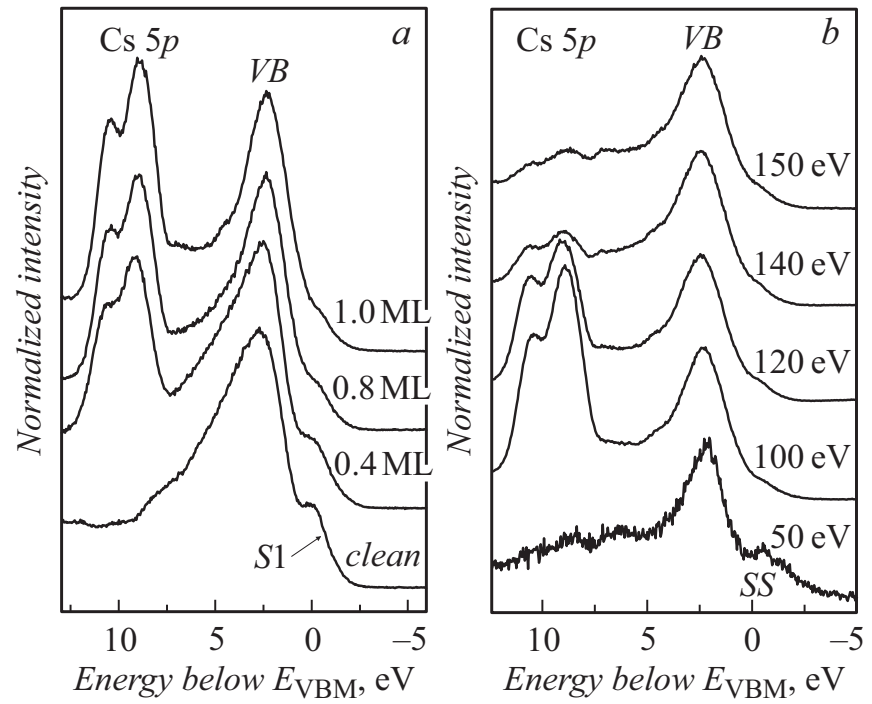

Рис. 2. $a-$ нормированные спектры фотоэмиссии для $n$ - $\mathrm{GaN}(0001)$ при различных покрытиях цезия. Энергия возбуждения $h v=120 \mathrm{eV}, b-$ спектры нормальной фотоэмиссии в области валентной зоны для наноинтерфейса $\mathrm{Cs} / n-\mathrm{GaN}(0001)$ при цезиевом покрытии $1.0 \mathrm{ML}$ и различных энергиях возбуждения.

валентной зоны на поверхности $E_{\mathrm{VBM}}$, которая определяется линейной аппроксимацией низкоэнергетического края спектра. Фон вычитался по методу Ширли. В области валентной зоны спектр исходного образца $n$ - $\mathrm{GaN}(0001)$ представляет собой слабоструктурированную полосу фотоэмиссии шириной $\sim 9 \mathrm{eV}$. Форма спектров незначительно зависит от энергии возбуждения. Единственная явная особенность $S 1$ наблюдается вблизи $E_{\mathrm{VBM}}$ в запрещенной зоне и означает металлизацию поверхности, которую можно связать с наличием атомов галлия в поверхностном слое. В расчетах $[1,8]$ показано, что поверхностное состояние $S 1$ существует только для поверхности $n-\mathrm{GaN}(0001)-2 \times 2$ и его природа связана с оборванными связями $\mathrm{Ga}$. Полученные нами спектры находятся также в хорошем согласии с экспериментальными данными $[1,14]$. 
Адсорбция Cs в субмонослойном режиме на $n$ - $\mathrm{GaN}(0001)$ не вызывает существенных изменений в спектрах фотоэмиссии. На рис. 2, $a$ представлены спектры фотоэмиссии, нормированные на максимальную интенсивность, при энергии возбуждения $h v=120 \mathrm{eV}$. Обнаружено, что адсорбция Cs практически полностью подавляет фотоэмиссию из пика поверхностного состояния $S 1$ и происходит уменьшение ширины валентной зоны при увеличении Cs-покрытия от 0.4 до $0.8 \mathrm{ML}$. При дальнейшем увеличении покрытия Cs форма спектра валентной зоны не изменяется. Такое поведение можно связать с подавлением поверхностных состояний $\mathrm{GaN}(0001)$, которые расположены при энергиях связи, бо́льших чем $3 \mathrm{eV}$. Подавление интенсивности поверхностного состояния $S 1$ ранее наблюдалось при адсорбции кислорода и воды на $\mathrm{GaN}(0001)-2 \times 2$ [1,2]. Полученные нами результаты хорошо согласуются с расчетом электронной структуры $\mathrm{Cs} / \mathrm{GaN}(0001)-2 \times 2$ для $\theta=0.33 \mathrm{ML}[10]$.

Спектры фотоэмиссии в области валентной зоны для $\mathrm{Cs} / n-\mathrm{GaN}(0001)$ при цезиевом покрытии $1.0 \mathrm{ML}$ подробно представлены на рис. $2, b$ в диапазоне энергий возбуждения $50-150 \mathrm{eV}$. Так же как и для чистой поверхности $\mathrm{GaN}$, спектры валентной зоны для разных энергий возбуждения практически совпадают. Интенсивность пика поверхностных состояний SS существенно уменьшается при энергиях возбуждения $h v>80 \mathrm{eV}$, а при меньших энергиях возбуждения $h v<60 \mathrm{eV}$ этот пик явно виден. У нового пика $S S$ спектральная зависимость от энергии возбуждения (рис. $2, b$ ) существенно отличается от аналогичной зависимости для пика $S 1$ (рис. 1), которая является значительно более широкой. Дополнительным аргументом в пользу того, что пик $S S$ связан с адсорбцией Cs является тот факт, что глубина выхода фотоэлектронов при уменьшении энергии возбуждения уменьшается, а при $h v=50 \mathrm{eV}$ она является минимальной, что указывает на локализацию данного состояния на поверхности. Ранее появление пика около уровня Ферми, индуцированного адсорбцией атомов Cs, найдено в [15]. Поэтому можно сделать вывод, что зона собственных поверхностных состояний подложки $S 1$ подавляется, а вместо нее формируется новая зона Cs-состояний $S S$. Можно предположить, что в ней не участвуют $p$-состояния Cs, так как наблюдаемая спектральная зависимость возбуждения Cs $5 p$-дублета (рис. $2, b)$ имеет максимум при энергиях возбуждения $h v=100-120 \mathrm{eV}$, и поэтому, скорее всего, новая зона сформирована в основном $s$-состояниями Cs.

Письма в ЖТФ, 2018, том 44, вып. 6 

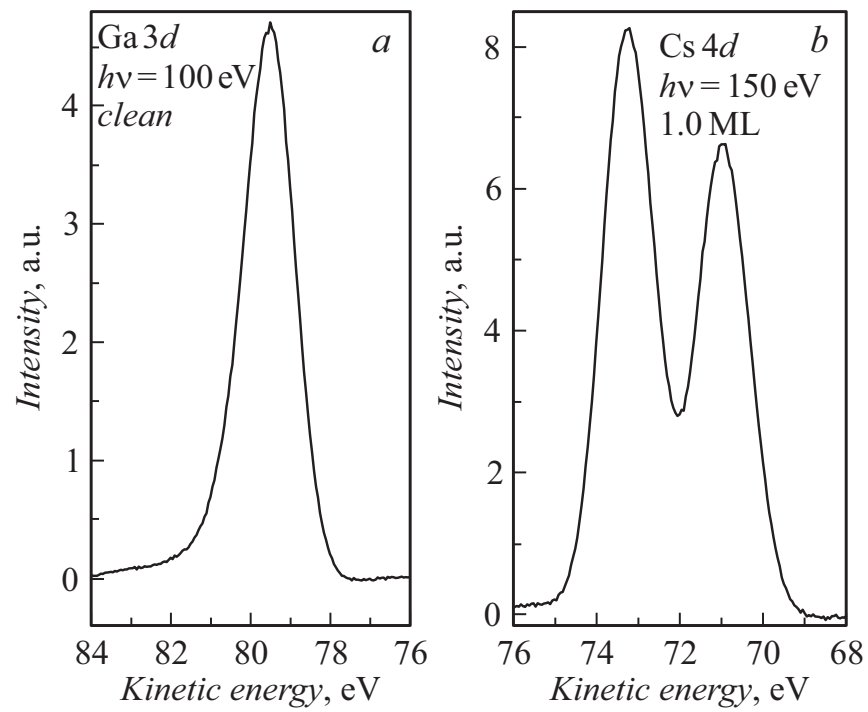

Рис. 3. Спектры нормальной фотоэмиссии остовного уровня $\mathrm{Ga} 3 d$ для чистой поверхности $n-\mathrm{GaN}(0001)$ при энергии возбуждения $h v=100 \mathrm{eV}(a)$ и остовного уровня $\mathrm{Cs} 4 d$ для интерфейса $\mathrm{Cs} / n-\mathrm{GaN}(0001)$ при цезиевом покрытии 1.0 ML и энергии возбуждения $h v=150 \mathrm{eV}(b)$.

На рис. 3, $a$ представлена фотоэмиссия из остовного уровня $\mathrm{Ga} 3 d$ для чистой поверхности $\mathrm{GaN}$ при энергии возбуждения $100 \mathrm{eV}$. На pис. $3, b$ показан дублет остовного уровня Cs $4 d$ при покрытии Cs $1.0 \mathrm{ML}$ на поверхности $\mathrm{GaN}$, энергия возбуждения $150 \mathrm{eV}$. Исследование фотоэмиссии из остовных уровней $\mathrm{Ga} 3 d$ и Cs $4 d$ свидетельствует о том, что форма остовных пиков при адсорбции Cs не изменяется, а происходит уменьшение интенсивности пика Ga $3 d$ в 2 раза и рост интенсивности дублета Cs $4 d$.

Исследована адсорбция Сs на поверхности $n-\mathrm{GaN}(0001)$ в субмонослойном режиме и в широком диапазоне энергий возбуждения. Найдено, что чистая поверхность $n-\mathrm{GaN}(0001)$ и наноинтерфейс $\mathrm{Cs} / n$ $\mathrm{GaN}(0001)$ проявляют металлический характер. Обнаружена эволюция спектра поверхностных состояний вблизи максимума валентной зоны при адсорбции Cs. 
Авторы благодарят Г.Э. Франк-Каменецкую и Н.М. Шмидт за помощь в экспериментальных исследованиях. Авторы выражают благодарность центру Гельмгольца в Берлине за предоставление возможности проведения экспериментов на синхротроне BESSY II и сотрудникам российско-германского канала за помощь во время подготовки и проведения экспериментальных исследований.

\section{Список литературы}

[1] Lorenz P., Gutt R., Haensel T., Himmerlich M., Schaefer J.A., Krischok S. // Phys. Status Solidi C. 2010. V. 7. N 2. P. 169-172.

[2] Widstrand S.M., Magnusson K.O., Johansson L.S.O., Oshima M. // Surf. Sci. 2005. V. 584. N 2-3. P. 169-178.

[3] Dhesi S.S., Stagarescu C.B., Smith K.E., Doppalapudi D., Singh R., Moustakas T.D. // Phys. Rev. B. 1997. V. 56. N 16. P. 10271-10275.

[4] Himmerlich M., Lymperakis L., Gutt R., Lorenz P., Neugebauer J., Krischok S. // Phys. Rev. B. 2013. V. 88. N 12. P. 125304(1-7).

[5] Grodzicki M., Mazur P., Zuber S., Brona J., Ciszewski A. // Appl. Surf. Sci. 2014. V. 304. P. 20-23.

[6] Chao Y.-C., Stagarescu C.B., Downes J.E., Ryan P., Smith K.S., Hanser D., Bremser M.D., Davis R.F. // Phys. Rev. B. 1999. V. 59. N 24. P. R15586R15589.

[7] Strasser T., Solterbeck C., Starrost F., Schattke W. // Phys. Rev. B. 1999. V. 60. N 16. P. 11577-11585.

[8] Segev D., Van de Walle C.G. // J. Cryst. Growth. 2007. V. 300. N 1. P. 199-203.

[9] Wang F.-H., Krüger P., Pollmann J. // Phys. Rev. B. 2001. V. 64. N 3. P. 035305(1-8).

[10] Du Y., Chang B., Wang X., Zhang J., Li B., Wang M. // Appl. Surf. Sci. 2012. V. 258. N 19. P. 7425-7429.

[11] Ptasinska M., Soltys J., Piechota J., Krukowski S. // Vacuum. 2014. V. 99. P. 166-174.

[12] Wu C.I., Kahn A. // Appl. Surf. Sci. 2000. V. 162-163. P. 250-255.

[13] Machuca F., Sun Y., Liu Z., Ioakeimidi K., Pianetta P., Pease R.F.W. // J. Vac. Sci. Technol. B. 2000. V. 18. N 6. P. 3042-3046.

[14] Kampen T.U., Eyckeler M., Moench W. // Appl. Surf. Sci. 1998. V. 123-124. P. 28-32.

[15] Benemanskaya G.V., Lapushkin M.N., Timoshnev S.N. // Surf. Sci. 2009. V. 603. P. 2474-2478.

Письма в ЖТФ, 2018, том 44, вып. 6 
[16] Ji Y., Du Y., Wang M. // Sci. World J. 2014. V. 2014. P. 490853 (1-6).

[17] Chugh M., Ranganathan M. // J. Phys. Chem. C. 2016. V. 120. N 15. P. 8076 8086.

[18] Fedorus A.G., Naumovets A.G., Vedula Yu.S. // Phys. Status Solidi A. 1972. V. 13. N 2. P. $445-456$. 